\section{Enterococcus raffinosus infection with atypical hemolytic uremic syndrome in a multiple myeloma patient after autologous stem cell transplant}

\author{
Pankaj Mathur, ${ }^{1}$ Blake Hollowoa, ${ }^{2}$ \\ Nupur Lala, ${ }^{2}$ \\ Sharmilan Thanendrarajan, ${ }^{1}$ \\ Aasiya Matin, ${ }^{1}$ Atul Kothari, ${ }^{1}$ \\ Carolina Schinke ${ }^{1}$ \\ ${ }^{1}$ Myeloma Institute, University of \\ Arkansas Medical Sciences, Medical \\ Center, Little Rock, AR; ${ }^{2}$ MS4 \\ University of Arkansas Medical \\ Sciences, Little Rock, AR, USA
}

\begin{abstract}
Autologous hematopoietic stem cell transplant (AHSCT) is the standard of care in the treatment of multiple myeloma worldwide. Infections are one of the most common complications of the chemotherapy regimen and AHSCT. Thrombotic microangiopathies are one of the rare but potentially life-threatening complications of infections associated with AHSCT. Thrombotic thrombocytopenic purpura and hemolytic uremic syndrome (HUS) are two most common type of thrombotic microangiopathies. The HUS is classically related to diarrheal illness such as with E.coli strain O157: H7 that produce Shiga-like toxins. But it has never been described with Enterococcus raffinosus urinary tract infections (UTI). Here we are describing a case of atypical HUS associated with Enterococcus raffinosus UTI in a patient with multiple myeloma after AHSCT. The management of atypical HUS especially after AHSCT is challenging. Eculizumab, a humanized monoclonal antibody against complement protein $\mathrm{C} 5$, and thrombomodulin have emerging role in the management of some cases, but more studies are needed to define evidence-based management of this condition.
\end{abstract}

\section{Introduction}

Autologous hematopoietic stem cell transplant (AHSCT) is the standard of care in the treatment for multiple myeloma worldwide. Infections are commonly associated with the chemotherapy regimen and AHSCT. Thrombotic microangiopathies such as atypical HUS are rare but potentially life-threatening complications of infections associated with AHSCT leading to increased morbidity and mortality after stem cell transplant. Atypical HUS is caused by endothelial toxicity related to chemotherapeutic agents and infections. The complement activation and dysregulation leads to the clinical hallmarks of hemolytic anemia and thrombocytopenia that are also seen in other thrombotic microangiopathies. Plasmapheresis, intravenous immunoglobulins (IVIG) and steroids have been used with variable success. The C5 complement antibody eculizumab, as well as thrombopoietin agonists, are new emerging agents which have been successfully used in some studies. Here we are describing a case of atypical HUS associated with Enterococcus raffinosus UTI in a multiple myeloma patient after AHSCT.

\section{Case Report}

The patient is a 62 y.o. Female, with past medical history of hypertension and gastroesophageal reflux disease, was diagnosed with Ig G-lambda multiple myeloma with initial presentation of acute renal insufficiency. Her bone marrow had $60 \%$ plasma cells on bone marrow aspirate at the time of diagnosis. She was treated with VDT-ACE (bortezomib, dexamethasone, thalidomide, adriamycin, cyclophosphamide, etoposide) induction chemotherapy and her renal function normalized. Repeat bone marrow showed 5\% plasma cells on aspirate. The second induction chemotherapy cycle was done with VDTPACE (bortezomib, dexamethasone, thalidomide, adriamycin, cyclophosphamide, etoposide, cisplatin) with stem cell mobilization collection. After the second induction cycle of chemotherapy, her bone marrow was negative for plasma cells. For consolidation phase, she received Melphalan $200 \mathrm{mg} / \mathrm{m}^{2}$ based autologous stem cell transplant as an outpatient. Subsequently, she was admitted to the hospital for neutropenic fever with severe mucositis, diarrhea and Enterococcus raffinosus associated urinary tract infection.

The patient was admitted to the hospital. The testing for infectious causes including HHV6/HHV8, HSV1/2, EBV, CMV, adenovirus, parvovirus and stool for $C$. difficile was negative. The patient was started on broad-spectrum antibiotics and antifungals imipenem, vancomycin, and micafungin. Repeated blood cultures and urine cultures were negative, and antibiotics were
Correspondence: Pankaj Mathur, Myeloma Institute, University of Arkansas Medical Sciences, Medical Center, Slot 816, Little Rock Arkansas, 72205 AR, USA.

E-mail : pmathur@uams.edu

Key words: Multiple myeloma, autologous stem cell transplant, transplant associated microangiopathy.

Contributions: the authors contributed equally.

Conflict of interest: the authors declare no potential conflict of interest.

Received for publication: 15 February 2017.

Revision received: 18 August 2017.

Accepted for publication: 18 August 2017.

This work is licensed under a Creative Commons Attribution-NonCommercial 4.0 International License (CC BY-NC 4.0).

(C) Copyright P. Mathur et al., 2017

Licensee PAGEPress, Italy

Hematology Reports 2017; 9:7094

doi:10.4081/hr.2017.7094

stopped after completion their course of two weeks.

During her hospital course, she developed hypertension, acute renal insufficiency with elevated creatinine, LDH and liver enzymes including bilirubin (Figure 1). In the peripheral smear, she had features of microangiopathy including thrombocytopenia, hemolytic anemia with schistocytes $>6$ / HPF. The patient became drowsy, though afebrile was transferred to intensive care unit for suspicion of thrombotic thrombocytopenic purpura (TTP). She was also started on plasmapheresis as the clinical features were suggestive of TTP. However her plasma ADAMTS 13 level was around 46\%. Therefore classical TTP was excluded, and plasmapheresis after three sessions was stopped. The autoimmune profile including ANA, ds DNA was negative. The complements C3, C4 were within normal limits, but $\mathrm{CH} 50$ levels were elevated.

The patient engrafted, recovered WBC counts, but microangiopathic hemolytic anemia with thrombocytopenia persisted. We made a working diagnosis of atypical HUS associated with Enterococcus raffinosus UTI after AHSCT in a patient with multiple myeloma. The gene rearrangement for atypical HUS was negative though as mentioned in the discussion below, it is positive only in approximately 50\% patients with atypical HUS. Among other coagulation parameters, direct coombs test was negative, coagulation profile PT/APTT, INR was within normal limits, however, d- 
dimers were persistently high, and fibrinogen levels continue to be on lower limits of normal. Since atypical HUS has a complement mediated autoimmune pathology, it was decided to give her eculizumab. Meanwhile pending approval of this medication, we decided to give her high dose IVIG $(0.5 \mathrm{~g} / \mathrm{kg} \times 3$ days followed by $1 \mathrm{~g} / \mathrm{kg}$ $\times 3$ days) in combination with $1 \mathrm{mg} / \mathrm{kg}$ prednisone for the underlying autoimmune pathology of the disease. The liver function tests and LDH came down drastically after initiation of IVIG and prednisone, however, her thrombocytopenia and hemolytic anemia persisted. Subsequently, she was started on eculizumab. She received the meningococcal vaccine before starting eculizumab. She received three weekly doses of eculizumab, along with ciprofloxacin prophylaxis. Her hemolytic anemia improved after eculizumab but her thrombocytopenia persisted. The repeat bone marrow examination showed decreased megakaryocytes. She was started on eltrombopag. Her platelets levels stabilized and subsequently discharged home (Figure 2).

\section{Discussion and Conclusions}

Hemolytic uremic syndrome (HUS) is a rare thrombotic microangiopathy characterized by microangiopathic hemolytic anemia, thrombocytopenia, and renal injury. ${ }^{1}$ The typical HUS primarily affects children. ${ }^{2}$ Approximately $90 \%$ of cases are preceded by an Escherichia coli infection, typically with E.coli strain O157:H7 that produce Shiga-like toxins and is thus classified as Shiga toxin-induced HUS (STECHUS). ${ }^{1,2}$ Typical HUS is generally associated with a good prognosis and low mortality. Atypical HUS also has features of microangiopathic hemolytic anemia, thrombocytopenia, and renal injury. Atypical HUS is associated with increased mortality, and about $50-60 \%$ of cases progress to endstage renal disease. ${ }^{1}$ The thrombotic microangiopathies occurring after hematopoietic stem cell transplant are particularly devastating and have very high mortality rates. ${ }^{2,3}$

In our patient, clinical features were suggestive for atypical HUS with Enterococcus raffinosus UTI after AHSCT for multiple myeloma. The species Enterococcus raffinosus was first recognized in $1989,{ }^{4}$ since then it has been associated with various infections in immunosuppressed patients especially in the acute care hospital settings. ${ }^{4,5}$ The natural habitat of Enterococcus raffinosus is unknown, but the organism has been found in the oropha- ryngeal flora of cats. It has been associated with wound infections, abscesses, urinary tract infections, vertebral osteomyelitis and endocarditis. ${ }^{4-9}$ However, microangiopathic hemolytic anemia has never been described in the literature with Enterococcus UTI. Though hemolytic uremic syndrome has been described with urinary tract infections with E. coli. ${ }^{10,11}$

Chiurchiu et al. ${ }^{12}$ first reported that hemolytic uremic syndrome could be associated with non-diarrheal Shiga toxin producing Escherichia coli O157:H7 which causes bacteremia and urinary tract infection. Later, Park et al. ${ }^{13}$ studied the association between thrombotic microangiopathy and UTI. They found out that $23 \%$ of the visits for thrombotic thrombocytopenic purpura had UTI and concluded that occult bacterial infections could cause alterations

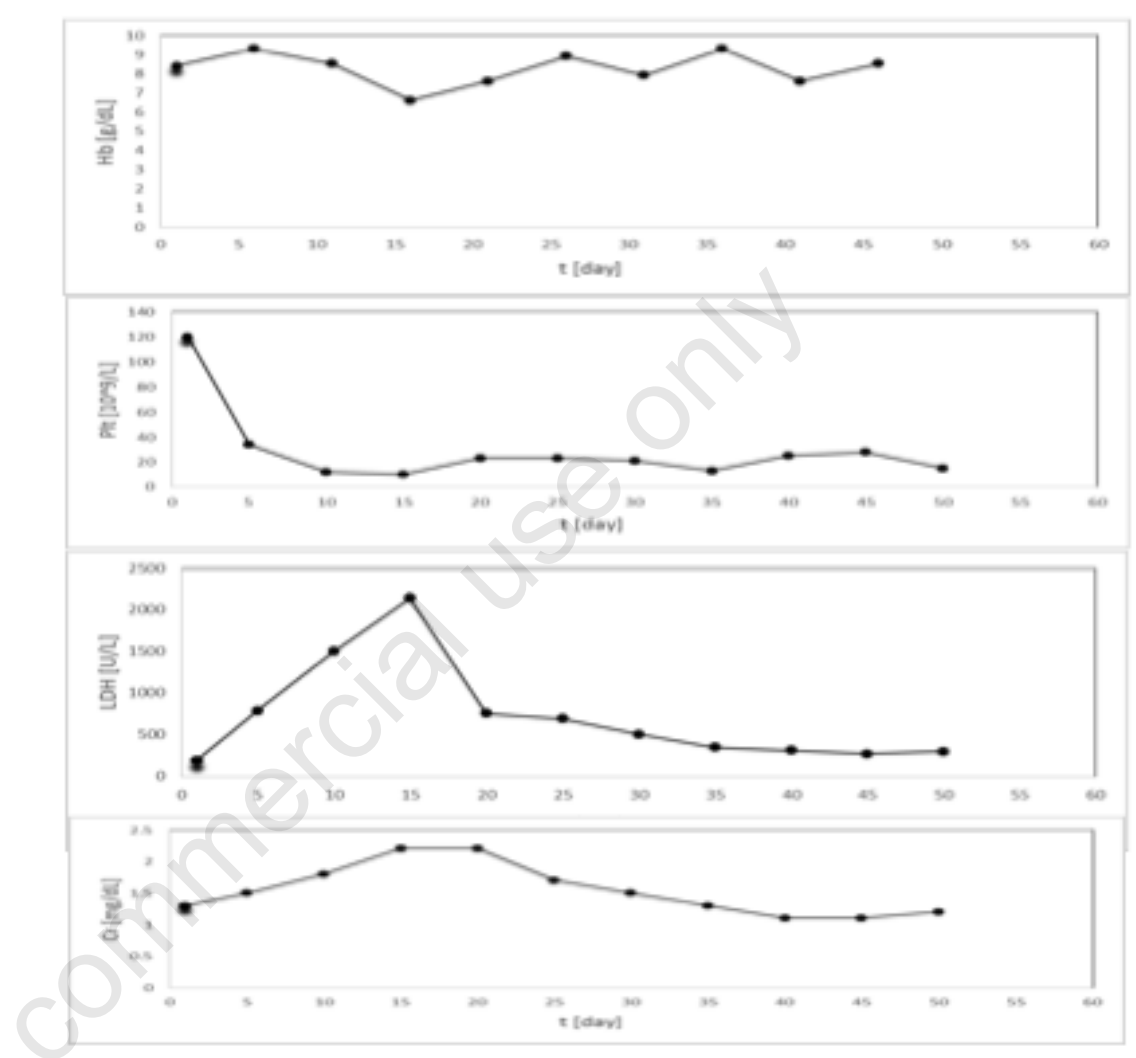

Figure 1. The graph showing the laboratory results in the patient with multiple myeloma with Enterococcus raffinosus infection

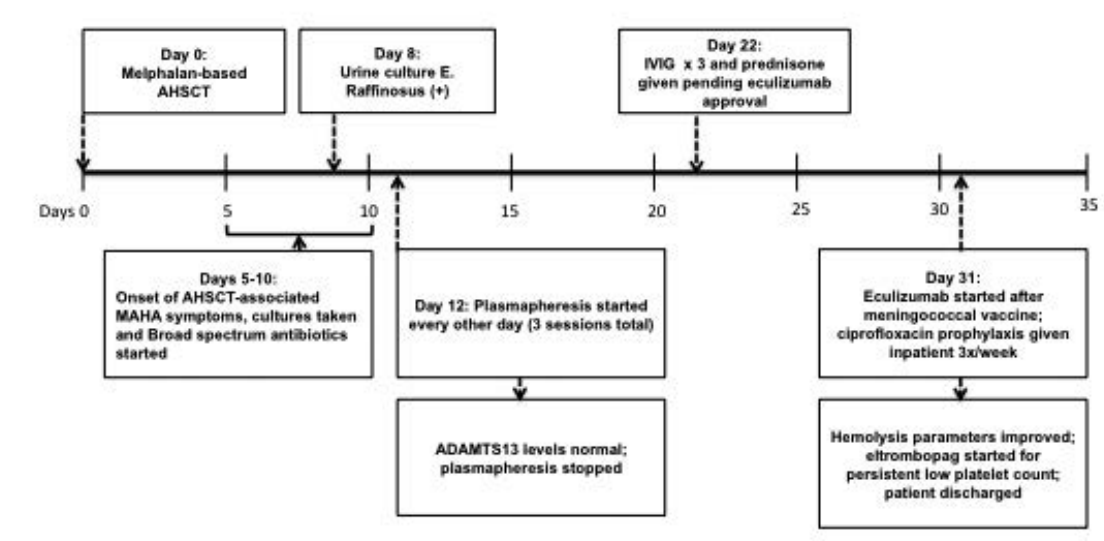

Figure 2. Timeline of the atypical HUS after Enterococcus raffinosus UTI infection after AHSCT in a multiple myeloma patient. 
Classical and alternative complement activation pathways

Triggers for the alternative pathway include Bacterial cell walls, lipopolysaccharide Eungal cell walls, Virally infected cells

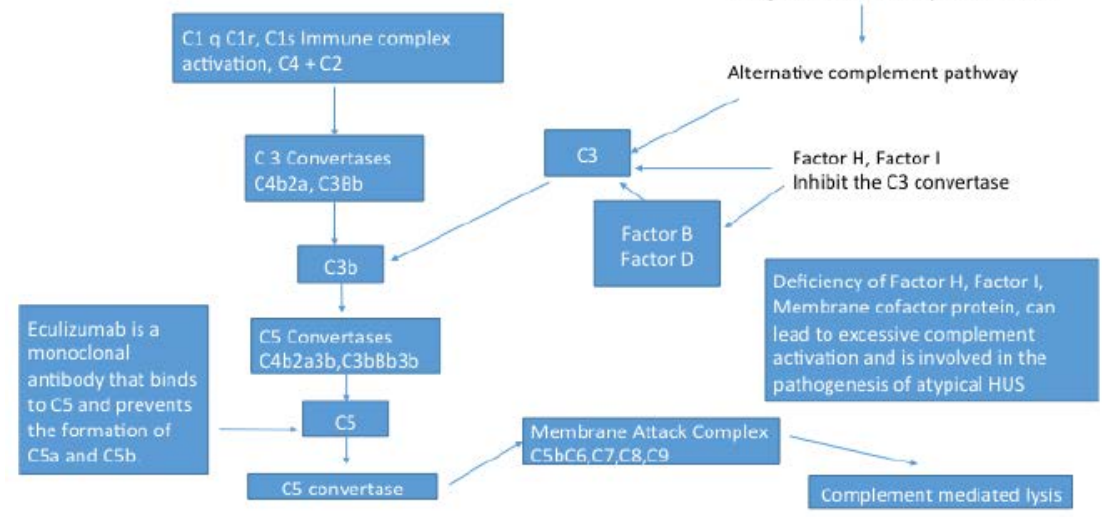

Figure 3. Classical and alternative pathways and role in pathogenesis of atypical HUS.

in the coagulation pathways probably resulting from the molecular mimicry between antibodies directed against infectious agents and the ADAMTS13 protein moiety. ${ }^{12-14}$

Over-activation of the complement system is the most common etiology of atypical HUS. ${ }^{14}$ The dysregulation of alternative complement pathway plays a central role in the pathogenesis of atypical HUS (Figure 3 ). The regulation of complement pathway is critical in preventing thrombosis from occurring, and genetic mutations have been described in up to $60 \%$ of adult aHUS case. ${ }^{14,15}$ The alteration in the alternative complement pathways especially mutations in complement factor I, complement factor $\mathrm{H}$, and membrane cofactor protein are among the commonly seen abnormalities and account for approximately 50\% of aHUS cases. ${ }^{14,15}$ Drugs such as calcineurin inhibitors and sirolimus have also been shown to cause endothelial injury and decrease VEGF expression leading to thrombotic microangiopathy. ${ }^{16}$ Sepsis may cause an alteration in ADAMTS13 activity most likely due to cleavage by circulating proteolytic enzymes. ${ }^{17}$

Until recently, treatment of aHUS was accomplished with plasma exchange although the efficacy of this treatment was variable and approximately $50 \%$ progressed to ESRD. ${ }^{18}$ Eculizumab, a monoclonal antiC5 component antibody, is a promising new treatment option for aHUS. ${ }^{19}$ Eculizumab has also shown to be useful in treating typical HUS caused by STEC and thrombotic thrombocytopenic purpura (TTP) in which ADAMTS13 levels are below acceptable ranges. ${ }^{20,21}$

Patients who develop atypical HUS or any thrombotic microangiopathy following
AHSCT have a high likelihood of succumbing to it and progression to multi-organ failure. This makes it especially important to identify candidates with the underlying genetic anomaly that predisposes them to this condition as well as the patients who will respond to eculizumab. Recently, recombinant human soluble thrombomodulin has also been used successfully to treat thrombotic microangiopathy after hematopoietic stem cell transplantation. ${ }^{22}$ Lastly, we need more studies to determine the exact pathophysiology of all these relatively uncommon disorders which can help proper diagnosis and management of these conditions especially in the setting of autologous stem cell transplants in hematological malignancies.

\section{References}

1. Conway EM. HUS and the case for complement. Blood 2015;126:2085-90.

2. Noris M, Mescia F, Remuzzi G. STECHUS, atypical HUS and TTP are all diseases of complement activation. Nat Rev Nephrol 2012;8:622-33

3. Chapin J, Shore T, Forsberg P, et al. Hematopoietic transplant-associated thrombotic microangiopathy: case report and review of diagnosis and treatments. Clin Adv Hematol Oncol 2014;12:565-73.

4. Chirurgi VA, Oster SE, Goldberg AA, et al. Ampicillin-resistant Enterococcus raffinosus in an acute-care hospital: case-control study and antimicrobial susceptibilities. J Clin Microbiol 1991;29: 2663-5.

5. Freyaldenhoven BS, Schlieper G,
Lütticken R, Reinert RR. Enterococcus raffinosus infection in an immunosuppressed patient: case report and review of the literature. J Infect 2005;51:e1214.

6. Savini V, Manna A, D'Antonio F, et al. First report of vaginal infection caused by Enterococcus raffinosus. J Med Microbiol 2008;57:672-3.

7. Mastroianni A. Enterococcus raffinosus endocarditis. First case and literature review. Infez Med 2009;17:14-20.

8. Prakash VP, Rao SR, Parija SC. Emergence of unusual species of enterococci causing infections, South India. BMC Infect Dis 2005;5:14.

9. Sandoe JA, Witherden IR, Settle C. Vertebral osteomyelitis caused by Enterococcus raffinosus. J Clin Microbiol 2001;39:1678-9.

10. Tarr PI, Fouser LS, Stapleton AE, et al. Hemolytic-uremic syndrome in a sixyear-old girl after a urinary tract infection with Shiga-toxin-producing Escherichia coli O103:H2. N Engl J Med 1996;335:635-8.

11. Scheutz F, Olesen B, Nørgaard A. Two cases of human urinary tract infection complicated by hemolytic uremic syndrome caused by verotoxin-producing Escherichia coli. Clin Infect Dis 2000;31:815-6.

12. Chiurchiu C, Firrincieli A, Santostefano $\mathrm{M}$, et al. Adult nondiarrhea hemolytic uremic syndrome associated with Shiga toxin Escherichia coli O157:H7 bacteremia and urinary tract infection. Am J Kidney Dis 2003;41:E4.

13. Park YA, Schultz EF, Hay SN, Brecher ME. Thrombotic thrombocytopenic purpura and urinary tract infections: is there a connection? Am J Clin Pathol 2011;135:85-8.

14. Maga TK, Nishimura CJ, Weaver AE, et al. Mutations in alternative pathway complement proteins in American patients with atypical hemolytic uremic syndrome. Hum Mutat 2010;31:1445.

15. Noris M, Mescia F, Remuzzi G. STECHUS, atypical HUS and TTP are all diseases of complement activation. Nat Rev Nephrol 2012;8:622-33.

16. Sartelet H, Toupance O, Lorenzato M, et al. Sirolimus-induced thrombotic microangiopathy is associated with decreased expression of vascular endothelial growth factor in kidneys. Am J Transplant 2005;5:2441-7.

17. Ono T, Mimuro J, Madoiwa S, et al. Severe secondary deficiency of von Willebrand factor-cleaving protease (ADAMTS13) in patients with sepsisinduced disseminated intravascular coagulation: its correlation with devel- 
opment of renal failure. Blood 2006;107:528-34.

18. Loirat C, Frémeaux-Bacchi V. Atypical hemolytic uremic syndrome. Orphanet J Rare Dis 2011;6:60.

19. Legendre CM, Licht C, Muus P, et al. Terminal complement inhibitor eculizumab in atypical hemolytic-uremic syndrome. $\mathrm{N}$ Engl $\mathrm{J}$ Med
2013;368:2169-81.

20. Lapeyraque AL, Malina M, FremeauxBacchi V, et al. Eculizumab in severe Shiga-toxin-associated HUS. N Engl J Med 2011;364:2561-3.

21. Chapin J, Weksler B, Magro C, Laurence J. Eculizumab in the treatment of refractory idiopathic thrombotic thrombocytopenic purpura. $\mathrm{Br} \mathrm{J}$
Haematol 2012;157:772-4.

22. Fujiwara H, Maeda Y, Sando Y, et al. Treatment of thrombotic microangiopathy after hematopoietic stem cell transplantation with recombinant human soluble thrombomodulin. Transfusion 2016;56:886-92 